\title{
EQUADIFF 6
}

\section{Avner Friedman}

Free boundary problems in fluid dynamics

In: Jaromír Vosmanský and Miloš Zlámal (eds.): Equadiff 6, Proceedings of the International Conference on Differential Equations and Their Applications held in Brno, Czechoslovakia, Aug. 26 - 30, 1985. J. E. Purkyně University, Department of Mathematics, Brno, 1986. pp. 17--22.

Persistent URL: http://dml.cz/dmlcz/700160

\section{Terms of use:}

(C) Masaryk University, 1986

Institute of Mathematics of the Academy of Sciences of the Czech Republic provides access to digitized documents strictly for personal use. Each copy of any part of this document must contain these Terms of use.

This paper has been digitized, optimized for electronic delivery and
stamped with digital signature within the project $D M L-C Z$ : The Czech
Digital Mathematics Library http://project.dml.cz




\section{FREE BOUNDARY PROBLEMS IN FLUID DYNAMICS}

A. FRIEDMAN

Northwestern University

Evanston, Illinois 60201, U.S.A.

The velocity potential of a 2-dimensional ideal incompressible and irrotational fluid satisfies $\Delta \phi=0$; further, Bernoulli's law $|\nabla \phi|^{2}+2 p=$ const. yields $|\nabla \phi|=$ const. on the (free) boundary of the fluid in contact with air. Since $\nabla \phi$ is tangential to the free boundary, the stream function $u$ (i.e., the harmonic conjugate of $\phi$ ) satisfies:

$$
\begin{aligned}
& \Delta u=0 \quad \text { in the fluid } \\
& u=c, \frac{\partial u}{\partial v}=\lambda \text { on the free boundary }
\end{aligned}
$$

where $c, \lambda$ are constants. If we take gravity into account, then $\lambda$ is replaced by $\sqrt{a+9 y}(a>0, g>0)$ where the gravitational force is in the upward direction.

In addition to (1) we must impose boundary conditions

$$
\begin{aligned}
& \mathrm{u}=\mathrm{u}_{0} \quad \text { or } \quad \frac{\partial \mathrm{u}}{\partial v}=\mathrm{u}_{1} \\
& \text { on the fixed boundary }
\end{aligned}
$$

as well as a condition at infinity. For example

(i) for a symmetric jet flow from a nozzle $l$ we have:

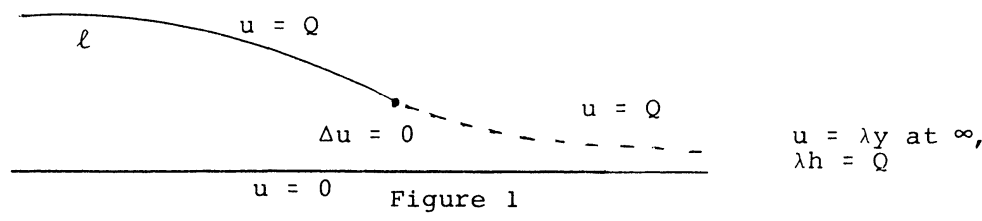

where $h$ is the asymprotic height of the free boundary as $x \rightarrow \infty$;

(ii) for a symmetric cavitational flow with nose $l$ we have:

$u>0$

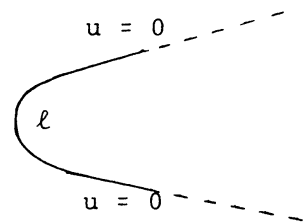

Figure 2 
$u=y(1+o(1)) \cdot 7 u=\vec{e}(1+o(1))$ where $\vec{e}=(0,1)$ and $o(1) \rightarrow 0$ if $x^{2}+y^{2} \rightarrow \infty$.

Problems such as (i), (ii) have been solved by several methods over the last 100 years. The general procedure has been to use conformal mappings or the hodograph transformation in order to reduce problems such as (i), (ii) to nonlinear integral equations (of a rather complicated type) and then apply the Leary-Schauder fixed point theorem; for details see [14][23] [24] and the references in [12], [22]. Another approach based on a variational principle was developed in [19], [20].

In the last few years Alt, Caffarelli and Friedman have developed a new variational approach to establish existence of solutions for free boundary problems. of general ideal fluids $[2-4,8,9]$, This work has also been extended to two fluids (flowing side-by-side)[2-4,89]. We shall explain the essence of the method in the simplest case (i) (Figure 1, above).

Consider the functional

where

$$
J(v)=\Omega_{\mu} \int\left|\nabla v-\lambda \vec{e} x_{\{v<Q\}} x_{E_{\mu}}\right|^{2} d x d y, \quad v \in K_{\mu}
$$

$$
\begin{aligned}
& \ell: y=g(x),-\infty<x<0, g \text { monotone }(b=g(0), A=(0, b)), \\
& E_{\mu}=\{(x, y) ;-\mu<x<\infty, 0<y<b\}, \Omega_{*}=\{(x, y) ; 0<y<g(x), \\
& \mathbf{R}_{+}^{2}=\{(x, y) ; x>0, y>0\} \text {, } \\
& \Omega=\Omega_{*} \cup \mathbf{R}_{+}^{2}, \Omega_{\mu}=\Omega \cap\{\mathrm{x}>-\mu\} \text {, } \\
& \mathrm{K}_{\mu}=\left\{\mathrm{v} \in \mathrm{H}^{1}\left(\mathbf{R}^{2}\right), \mathrm{v}=\mathrm{Q} \text { if } \mathrm{y} \geq \mathrm{g}(\mathrm{x}), \mathrm{x}<0 \text { or } \mathrm{y} \geq \mathrm{b}, \mathrm{x}>0\right. \text {; } \\
& \mathrm{v}(\mathrm{x}, 0)=0 \text { if }-\infty<\mathrm{x}<\infty \text {, } \\
& \mathrm{v}(-\mu, \mathrm{y})=\mathrm{h}_{\mu}(\mathrm{y}) \text {, and } 0 \leq \mathrm{v} \leq \mathrm{Q} \text { a.e.\}; }
\end{aligned}
$$

here $h_{\mu}(y)$ is a given function monotone in $y_{,} h_{\mu}(0)=0, h_{\mu}(g(-\mu))=Q$. Consider the problem: Find $v=v_{\lambda, \mu}$ in $k_{\mu}$ such that

$$
\min _{v \in K_{\mu}} J(v)=J\left(u_{\lambda, \mu}\right) \text {. }
$$

It is easily seen that this problem has a solution. The solution is harmonic in $\Omega_{*} \backslash E_{\mu}$ and is a local minimizer in $E_{\mu}$ of

$$
\tilde{J}(v) \equiv \int\left(|\nabla v|^{2}+\lambda^{2} x_{\{v<Q\}}\right) d x d y .
$$

Alt and Caffarelli [1] studied the local minimizer $\tilde{v}$ of $\tilde{J}$ and proved that $\widetilde{v}$ is Lipschitz continuous and that the free boundary $\partial\{\tilde{v}<Q\} \cap E_{\mu}$ is locally analytic. 
LEMMA 1. The minimizer is unique.

Indeed, suppose $u_{1}, u_{2}$ are two minimizers and introduce $u_{1}^{\varepsilon}(x, y)=u_{1}(x-\varepsilon, y)$ and

$$
v_{1}=u_{1}^{\varepsilon} \wedge u_{2}, \quad v_{2}=u_{1}^{\varepsilon} \vee u_{2} \text {. }
$$

Denote by $J^{\varepsilon}$ the functional $J=J_{\lambda, \mu}$ corresponding to the translation $\mathbf{x} \rightarrow \mathbf{x}+\varepsilon$ of $\Omega_{\mu}, \mathbf{K}_{\mu}$. One verifies that

$$
J^{\varepsilon}\left(u_{1}^{\varepsilon}\right)+J\left(u_{2}\right)=J^{\varepsilon}\left(v_{1}\right)+J\left(v_{2}\right),
$$

which implies that $J\left(u_{2}\right)=J\left(v_{2}\right)$, i.e., $u_{1}^{\varepsilon} v u_{2}$ is a minimizer. Consequently $u_{1}^{\varepsilon} \vee u_{2}$ is smooth, which implies that either $u_{1}^{\varepsilon} \geq u_{2}$ or $u_{1}^{\varepsilon} \leq u_{2}$ everywhere. Since $u_{1}^{\varepsilon}<u_{2}$ near the boundary, we deduce that $u_{1}^{\varepsilon}<u_{2}$ throughout the domain. Taking $\varepsilon \rightarrow 0$ we get $u_{1} \leq u_{2}$, and similarly $u_{2} \leq u_{1}$.

Taking $u_{1}=u_{2}$ in the above argument we get:

$$
\frac{\partial}{\partial x} u_{\lambda, \mu} \geq 0 \text {. }
$$

Thus the analytic free boundary $\Gamma=\Gamma_{\lambda, \mu}$ has the form

$$
\Gamma_{\lambda, \mu}: \mathbf{x}=\mathbf{f}_{\lambda, \mu}(\mathrm{y})
$$

Next we have:

LEMMA 2. $\mathrm{f}_{\lambda, \mu}(\mathrm{y})$ is continuous and finite if and only if $\mathrm{h}<\mathrm{y}<\mathrm{b}$, where $\mathrm{h}=\mathrm{Q} / \lambda$.

LEMMA 3. $\lambda \rightarrow f_{\lambda, \mu}(b)$ is continuous $\left(f_{\lambda, \mu}(b)=f_{\lambda, \mu}(b+0)\right)$.

LEMMA 4. If $\lambda$ is sufficiently small then $\mathrm{f}_{\lambda, \mu}(\mathrm{b})<0$; if $\lambda<Q / b$ and $|\lambda-Q / b|$ is small enough, then $f_{\lambda, \mu}(b)>0$.

From Lemmas 3,4 we deduce that there is a value $\lambda=\lambda(\mu)$ such that $\mathrm{f}_{\lambda, \mu}(\mathrm{b})=0$, i.e., there is a "continuous fit" at A. Fro this value of $\lambda,\left(u_{\lambda, \mu}, \Gamma_{\lambda, \mu}\right)$ "almost" solves the jet problem. In order to complete the construction of a solution we let $\mu \rightarrow \infty, \lambda(\mu) \rightarrow \lambda$ and denote the limiting $u_{\lambda, \mu}, \Gamma_{\lambda, \mu}$ by $u_{,} \Gamma$.

LEMMA 5. Continuous fit implies smooth fit. More precisely the curve $\ell \cup \Gamma$ is not only continuous at the 
point of detachment $A$, but it is also $C^{1}$ at $A$, and $\nabla u$ is uniformly $C^{1}$ in $\{u<Q\}-$ neighborhood of $A$.

THEOREM. There exists a unique classical solution of the symmetric jet problem (i).

Existence was already outlined above; uniqueness is proved by a comparison argument [21].

The above procedure has been extended to three-dimensional axially symmetric jets [2], 2-dimensional asymmetric flows [3], to flows in a gravity field [4], to rotational flows [16] and to compressible fluids [8] [9]; some cavity problems are treated in [13] [18].

Two-fluid problems are treated in [5-7]. Here $\mathrm{u}^{+}$and $\mathrm{u}^{-}$are harmonic and

$$
\frac{\partial u^{+}}{\partial v}-\frac{\partial u^{-}}{\partial v}=\lambda \text { on the free boundary. }
$$

In a two-fluid flow in a porous media of a rectangular dam, the free boundary condition can be reduced to

$$
\frac{\partial u^{+}}{\partial v}-\frac{\partial u^{-}}{\partial v}=\cos (x, v)
$$

which is similar to ( 3 ); in (3) $\lambda$ is not a priori given, whereas in (4) a degeneracy occurs at points where $\cos (x, v)=0$. Problem ( 4$)$ is studied in [10] where existence of a solution is proved having a $C^{1}$ free boundary.

Lemma 5 has been extended in [11] to quasilinear elliptic operators and to more general boundary conditions $\partial u / \partial \nu=f$. The assertion is that either $\Gamma \cup \ell$ is $C^{2}$ at $A$ or it is only in $c^{3 / 2}$ and the curvature of $\Gamma$ goes to $\pm \infty$ as $x \downarrow 0$.

Other physical flow problems lead to free boundary conditions as above. We mention the problem of freezing in a channel because of heat sink at the origin [25]. Thus

$$
\Delta \mathrm{u}=-\mathrm{M} \delta \text { in }\{\mathrm{u}>0\}
$$

where $\delta=$ Dirac measure, $-u$ is the temperature, and

$$
\begin{aligned}
& u>0 \text { in the ice, } \\
& \frac{\partial u}{\partial v}=\lambda \text { on the free boundary; }
\end{aligned}
$$

$\lambda$ and $M$ are given positive constants. Assuming that the channel $\Omega$ is 
symmetric with respect to teh $y$-axis it was recently proved by Friedman and 3 tojanovic [17] that the problem has a unique solution with free boundary concave to the ice. This implies that if $\partial \Omega$ consists of $p$ curves $l_{i}$ convex to $\Omega$ then the free boundary consists of at most $p$ arcs ("fingers") concave to $\Omega$, each connecting an adjacent pair $\ell_{i}$, $\ell_{i+1}$.

$R$ e $f$ e $r$ e e $s$

[1] H.W. Alt and A.Caffarelli, Existence and regularity for a minimum problem with free boundary. J. Reine Angew. Math. 105 (1981), $105-144$.

[2] H.W. Alt, L.A. Caffarelli and A. Friedman, Axially symmetric jet flows, Arch. Rat. Mech. Anal. 81 (1983), 97-149.

[3] H.W.Alt, L.A.Caffarelli and A. Friedman. Asymmetric jet flows, Comm. Pure Appl. Math. 35 (1982), 29 - 68.

[4] H.W.Alt, L.A.Caffarelli and A. Friedman, Jet flows with gravity, J. Reine Angew. Math. 331 (1982), 58-103,

[5] H.W.Alt, L.A. Caffarelli and A. Friedman, Variational problems with two phases and their free boundaries, Trans. Amer. Math. Soc., 282 (1984), 431-461.

[6] H.W.Alt, L.A.Caffarelli and A. Friedman, Jets with two fluids I: one free boundary, Indiana Univ. Math. J., 33 (1984), 213-247.

[7] H.W.Alt, L.A.Caffarelli and A. Friedman, Jets with two bluids II: two free boundaries, Indiana Univ. Math. J., 33 (1984), 367-391.

[8] H.W.Alt, L.A.Caffarelli and A. Friedman, A free boundary problem for quasi-linear elliptic equations, Ann. Sc. Norm. Sup. Pisa, $11(1984), 1-44$.

[9] H.W.Alt, L.A.Caffarelli and A. Friedman, Compressible flows of jets and cavities, J. Diff. Eqs., 56 (1985), 82-141.

[10] H.W.Alt, L.A.Caffarelli and A. Friedman, The dam problem with two fluids, Comm. Pure Appl. Math., 37 (1984), 601-646.

[11] H.W.Alt, L.A.Caffarelli and A. Friedman, Abrupt and smooth separation of free boundaries in flow problems, Scu. Norm. Sup. Pisa, to appear.

[12] G.D. Birkhoff and E.H. Zarantonello, Jets, wakes and cavities, Academic Press, New York, 1957.

[13] L.A. Caffarelli and A. Friedman, Axially symmetric in finite cavities, Indiana Univ. Math. J. 30 (1982), 135-160. 
[14] R. Finn, Some theorems on discontinuity of plane fluid motion, J.D'Analyse Math. 4 (1954/6), 246-29l.

[15] A. Friedman, Variational principles and free boundary problems, John Wiley, New York, 1982.

[16] A. Friedman, Axially symmetric cavities in rotational flows, Comm. in P.D.E., 8 (1983), 949-997.

[17] A. Friedman and S. Stojanovic, A free boundary problem associated with icing in a chanel, to appear.

[18] A. Friedman and T.I. Vogel, Cavitational flow in a chanel with oscillatory wall, Nonlinear Analysis, 7 (1983), 1157-1192.

[19] P.R. Garabedian, H. Lewy and M. Schiffer, Axially symmetric cavitational flow, Ann. of Math. 56 (1952), 560-602.

[20] P.R.Garabedian and D.C. Spencer, Extremal methods in cavitational flow, J. Rat. Mech. Anal. I (1952), 350-409.

[21] D. Gilbarg, Uniqueness of axially symmetric flows with free boundaries, J. Ra. Mech. Anal. I (1952), 309-320.

[22] D. Gilbarg, Jets and cavities, in Handbuch der Physik, vol. 9, Springer-Verlag New York, 1960.

[23] J. Leray, Les Problèmes de représentation conforme de Helmholtz I, II, Comm. Math. Helv. 8 (1935), 149-180; 250-263.

[24] J. Serrin, Existence theorems for some hydrodynamical free boundary porblems, J. Rat. Mech. Anal. I (1952), 1-48.

[25] L. Ting, D.S. Ahluwalia and M.J. Miksis, Solutions of a class of mixed free boundary problems, SIAM J. Appl. Math., 43 (1983), 759-775. 